\title{
ON THE DIFFUSION OF ALLOYING ELEMENTS IN THE NICKEL-BASE SUPERALLOYS
}

\author{
Chong Long Fu, Roger Reed*, Anderson Janotti and Maja Krcmar \\ Metals and Ceramics Division, Oak Ridge National Laboratory, PO Box 2008, TN 37830, USA \\ *Dept of Metals and Materials Engineering, \\ The University of British Columbia, 309-6350 Stores Road, Vancouver V6T 1Z4, Canada
}

Keywords: diffusion, diffusion mechanisms, quantum-mechanical modelling, alloy design

\begin{abstract}
The diffusion of transition metal solutes in nickel has been studied using quantum-mechanical first principles methods, and the predictions compared critically with the experimental data available in the literature. For the $4 \mathrm{~d}$ and $5 \mathrm{~d}$ rows which contain $\mathrm{Ru}$ and $\mathrm{Re}$ respectively, diffusion rates are largest for elements at the far west and far east of the d-block of transition metals. The calculations reveal that this is due to a significant barrier energy for solute-vacancy exchange for elements residing at the centre of the period, e.g. Ru and Re, despite their displaying atomic sizes which are closest to that of Ni. Thus it is demonstrated conclusively that the underlying electronic bonding controls the rate of diffusion. Elements such as Ru and Re are amongst the most dense and least compressible of the transition metals due to their configuration of electrons - when alloyed with $\mathrm{Ni}$ this causes directional and incompressible $\mathrm{Ni}-\mathrm{Ru}$ and $\mathrm{Ni}$-Re bonds to be formed which hinder vacancy migration. These effects dominate over any differences in the vacancy-solute binding energy and any influence of the atomic radius of the solute. In this respect, the results disprove the traditional view that diffusion of substitutional solutes is least rapid when the size misfit with the host is the greatest. The trends for the $3 \mathrm{~d}$ row are also studied, and it is shown that magnetism has a profound effect. Finally, if the theoretical results are to be rationalised with the experimental data, it is demonstrated
\end{abstract}

that the correlation factor - which accounts for diffusional jumps in the reverse direction - should be included in the model. The results explain why some elements - for example Re - have a strong effect on the high temperature properties of the superalloys, and provide insights for future alloy design efforts.

\section{Introduction}

The nickel-based superalloys are required to operate at elevated temperatures, often very close to their melting point. Under these conditions, a number of degradation mechanisms can arise which are usually mediated by diffusional processes. For example, creep deformation occurs at a rate dependent upon diffusional rearrangements at dislocation cores [1]. Directional coarsening of the $\gamma^{\prime}$ phase (the so-called rafting effect) requires mass transport on the scale of the periodicity of the $\gamma^{\prime}$ precipitates [2]. Single crystal superalloys can interdiffuse with coatings applied to them [3] and oxidation occurs at a rate which is diffusion-controlled [4]. Clearly, the retardation of diffusional processes is important if the very best properties are to be attained. Despite this fact, superalloy metallurgists know remarkably little about the rates of diffusion of the substitutional solutes added to their alloys. Indeed, the processes used to design new alloys still tend to be rather Edisonian in their approach, with considerable use made of trial-and-error procedures. 
However, recent work has quantified the rates of interdiffusion of the $4 \mathrm{~d}$ and $5 \mathrm{~d}$ transition metals in nickel $[5,6]$, see Figure 1, by analysing diffusion couples exposed to elevated temperatures. The concentration profiles obtained were measured using electron microprobe analysis and the interdiffusion coefficients deduced using the Sauer-Freise method.

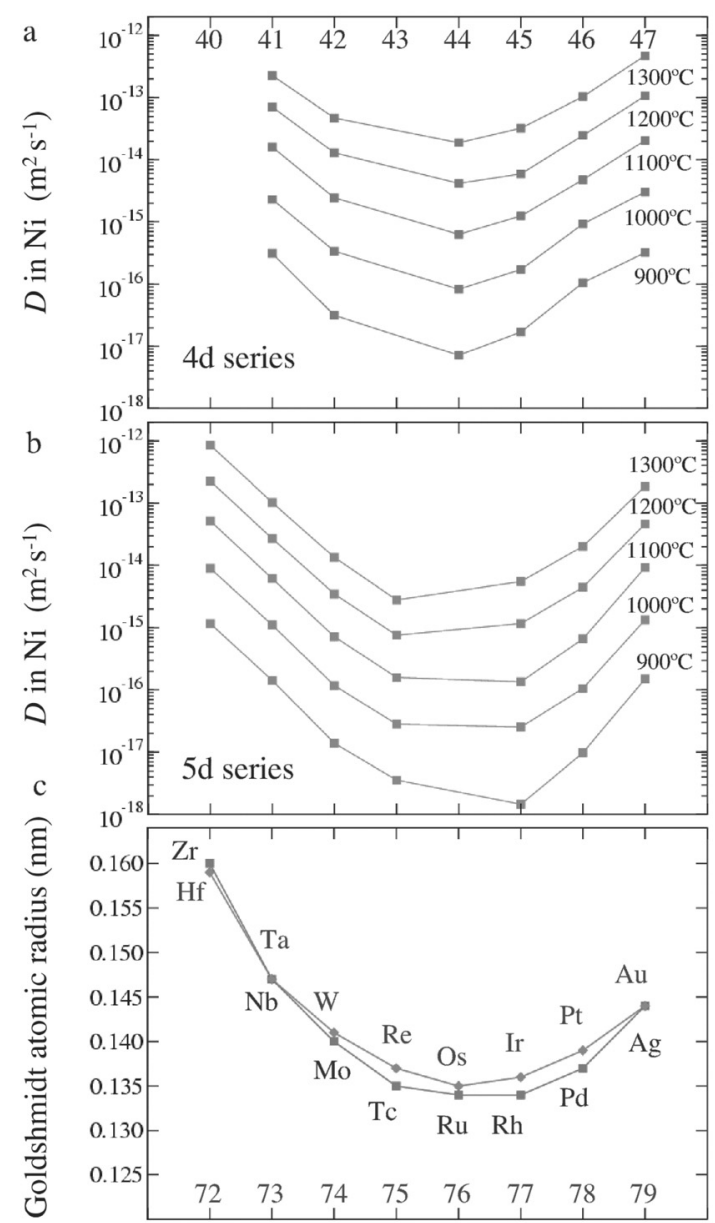

Figure 1: Variation of measured [5] interdiffusion coefficients with nickel for various substitutional solutes: (a) 4d row, (b) $5 \mathrm{~d}$ row and (c) corresponding variation of the Goldschmidt radii for the solutes. The atomic numbers of the $4 \mathrm{~d}$ and $5 \mathrm{~d}$ rows are given at the top and bottom of (a) and (c) respectively.

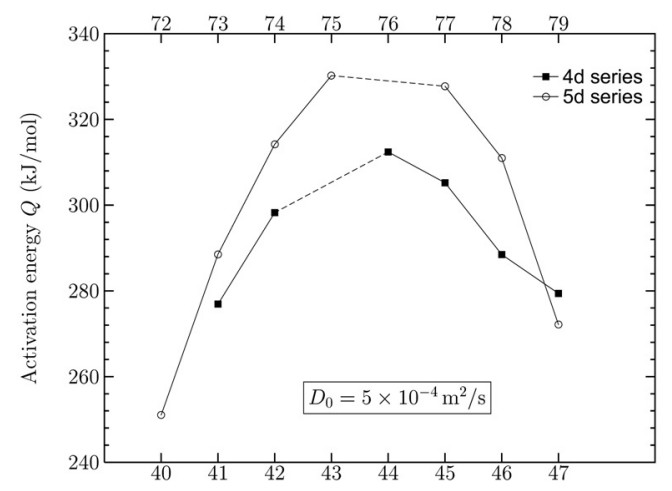

Figure 2: Variation of measured [5] activation energy for interdiffusion with $\mathrm{Ni}$, with the pre-exponential coefficient $D_{0}$ constrained to be $5 \times 10^{-4} \mathrm{~m}^{2} / \mathrm{s}$. Note that the correlation factor $f$ is taken to be unity.

A remarkable and surprising result was found: alloying additions taken from the middle of the transition metal block display the slowest rates of diffusion, despite exhibiting atom radii which are the closest to that of nickel $(0.125 \mathrm{~nm})$, see Figure 1. Elements from the far west and far east of the transition metal block exhibit the greatest rates of diffusion, although they possess the greatest lattice misfit with the nickel solvent. Both the second (4d) and third (5d) transition metal rows were found to display this effect. Moreover, it was suggested that this dependence resides in differences in the activation energy rather than the pre-exponential term, see Figure 2. Recently, it has been demonstrated that quantum-mechanical first principles methods are capable of rationalising these effects [7].

In [5], the first row (3d) transition metals were not considered. Indeed, it appears that no attempt has been made so far to identify a systematic correlation between the positions of the solutes in the d-block and the rates of interdiffusion with nickel. Here, this situation is remedied. To facilitate a critical evaluation of the modelling, various data have been collected and collated: for the interdiffusion of $\mathrm{Ti}$ [8], V [9], Cr [10], Mn [11], Fe [12], $\mathrm{Co}[10]$ and $\mathrm{Cu}[13]$ with the nickel host. Data for the self-diffusion of nickel are available in [14]. 


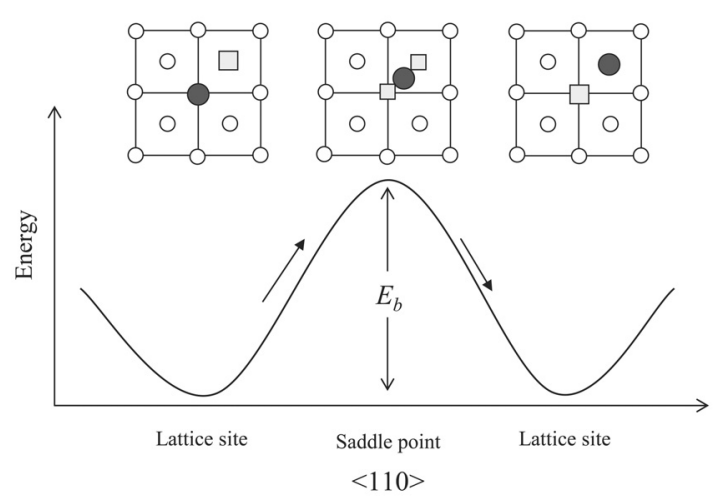

Figure 3: Definition of the diffusion energy barrier $E_{b}$, for vacancy displacement along the $\langle 110\rangle$ direction. Filled circles: solute atoms. Unfilled circles: solvent atoms. Squares: vacancies.

\section{Background}

The interaction of substitutional solutes with vacancies in a solvent such as nickel is in fact rather complicated [15]. According to the five-frequency exchange model developed by Lidiard $[15,16]$, five different atom-vacancy exchanges can be identified - each leading to a different local configuration of vacancy, solute and solvent atoms. However, to a first approximation it may be sensible to assume that the frequency $\Gamma_{s}$ of solute-vacancy exchanges controls the rate of interdiffusion, with its magnitude depending on a barrier energy $E_{b}$, see Figure 3. The diffusion coefficient $D$ is then given by [16]

$$
D=f a^{2} \Gamma_{s} p_{v}
$$

where

$$
\Gamma_{s}=\nu_{o} \exp \left\{-\frac{E_{b}}{k T}\right\}
$$

Here, $f$ is a correlation factor, $\nu_{o}$ is the Debye frequency and $a$ is the lattice parameter. The term $p_{v}$ is the probability that the site next to a solute atom is vacant; it depends upon the energy necessary to create a neighbouring vacancy, denoted $E_{f}^{V}$, such that $E_{f}^{V}=$
$E_{f}^{\mathrm{vac}, \mathrm{Ni}}+\Delta E_{V}^{*}$ where $E_{f}^{\mathrm{vac}, \mathrm{Ni}}$ is the vacancy formation energy in pure nickel and $\Delta E_{V}^{*}$ is the solute/vacancy binding energy. Thus

$$
p_{v}=C_{o} \exp \left\{-\frac{E_{f}^{V}}{k T}\right\}
$$

where $C_{o}$ is a constant. It follows that

$$
D=f a^{2} \nu_{o} \exp \left\{-\frac{E_{b}}{k T}\right\} \times C_{o} \exp \left\{-\frac{E_{f}^{V}}{k T}\right\}
$$

which has the familiar Arrhenius form

$$
D=f D_{o} \exp \left\{-\frac{Q}{k T}\right\}
$$

with $D_{o}$ the pre-exponential term and the activation energy being given by $Q=E_{b}+E_{f}^{V}$. Note that for reasons that will become apparent later in the paper, the correlation factor $f$ is not included in the pre-exponential term $D_{o}$. It is clear then that the activation energy for diffusion depends upon the sum of two terms, one corresponding to the barrier energy and the other the energy for the formation of a vacancy next to the solute atom.

\section{Computational Methods}

Quantum-mechanical first principles calculations were carried out using density functional theory within the local spin density approximation [17]. The total energy and forces were obtained by solving the local-densityfunctional equation using the ultra-soft pseudo-potential method $[18,19]$. The host nickel lattice was represented by a face-centered cubic (FCC) supercell consisting of 32 atoms, with one nickel atom replaced by a solute atom. For the estimation of the vacancy-solute interaction, a vacancy was introduced in a site next to the solute atom. To calculate the energy barrier for diffusion $E_{b}$, the solute atom was displaced towards the adjacent vacancy along the $\langle 110\rangle$ direction, the lattice vector relevant to diffusional flow, see Figure 3. At each point the position of the solute atom was fixed and all host $\mathrm{Ni}$ atoms were allowed to relax; the energy barrier is 
then the total energy difference for the solute atom at the saddle point and at the initial lattice site. To estimate the diffusion rate, the diffusion activation energy $Q$ was approximated as the sum of the diffusion energy barrier $E_{b}$ and the energy for formation of a vacancy next to a solute atom, denoted $E_{f}^{V}$. As a benchmark, the energy barrier for self-diffusion and the vacancy formation energy were determined for pure $\mathrm{Ni}$ : values of $120 \mathrm{~kJ} / \mathrm{mol}$ and $164 \mathrm{~kJ} / \mathrm{mol}$, respectively, were obtained. The calculated activation energy for self-diffusion is then $284 \mathrm{~kJ} / \mathrm{mol}$ which is in excellent agreement with the accepted value [14].

To estimate the pre-exponential term, transition state theory was employed: the migration entropy was estimated by determining the vibrational degrees of freedom associated with a collection of classical harmonic oscillators. Consistent with the available experimental data [5], these considerations suggest pre-exponential factors $D_{o}$ in the range $5 \times 10^{-5}$ to $1 \times 10^{-4} \mathrm{~m}^{2} / \mathrm{s}$, see [7].

\section{Results: 4d and 5d Rows}

Results for the calculated activation energies of diffusion for the $4 \mathrm{~d}$ and $5 \mathrm{~d}$ solutes in $\mathrm{Ni}$ are given in Figure 4. The predictions indicate that the activation energy does vary across the transition metal rows in an unexpected and counterintuitive way: larger atoms (elements at the far east and west of the d-block of elements) have lower activation energies than the smaller atoms (at the centre). The contributions from the vacancy formation energy and the diffusion energy barrier are shown in Figure $4 \mathrm{~b}$ and 4c. The vacancy formation energy displays the expected trend, i.e. the higher lattice strain induced by larger solute atoms such as Hf and Au causes $E_{f}^{V}$ to be lower for those elements. Given that the vacancy formation energy for pure $\mathrm{Ni}, E_{f}^{\mathrm{vac}, \mathrm{Ni}}$, is about $164 \mathrm{~kJ} / \mathrm{mol}$, one can see that the vacancy-solute binding energy $\Delta E_{V}^{*}$ varies from about $-10 \mathrm{~kJ} / \mathrm{mol}$ for Re to about $-40 \mathrm{~kJ} / \mathrm{mol}$ for $\mathrm{Hf}$ and $\mathrm{Au}$. However, the dependence of $E_{f}^{V}$ on atomic number is relatively weak; the major contribution to the variation of the activation energy $Q$ arises from the strong influence of the atomic number on the diffusion energy barrier $E_{b}$. Particularly notable is the prediction that the $Q$ 's are systematically greater for the $5 \mathrm{~d}$ row than for the $4 \mathrm{~d}$ one. This is consistent with the experimental values of the apparent activation energies - see Figure 2 - although in deriving these the reader should be aware that $f$ is taken to be

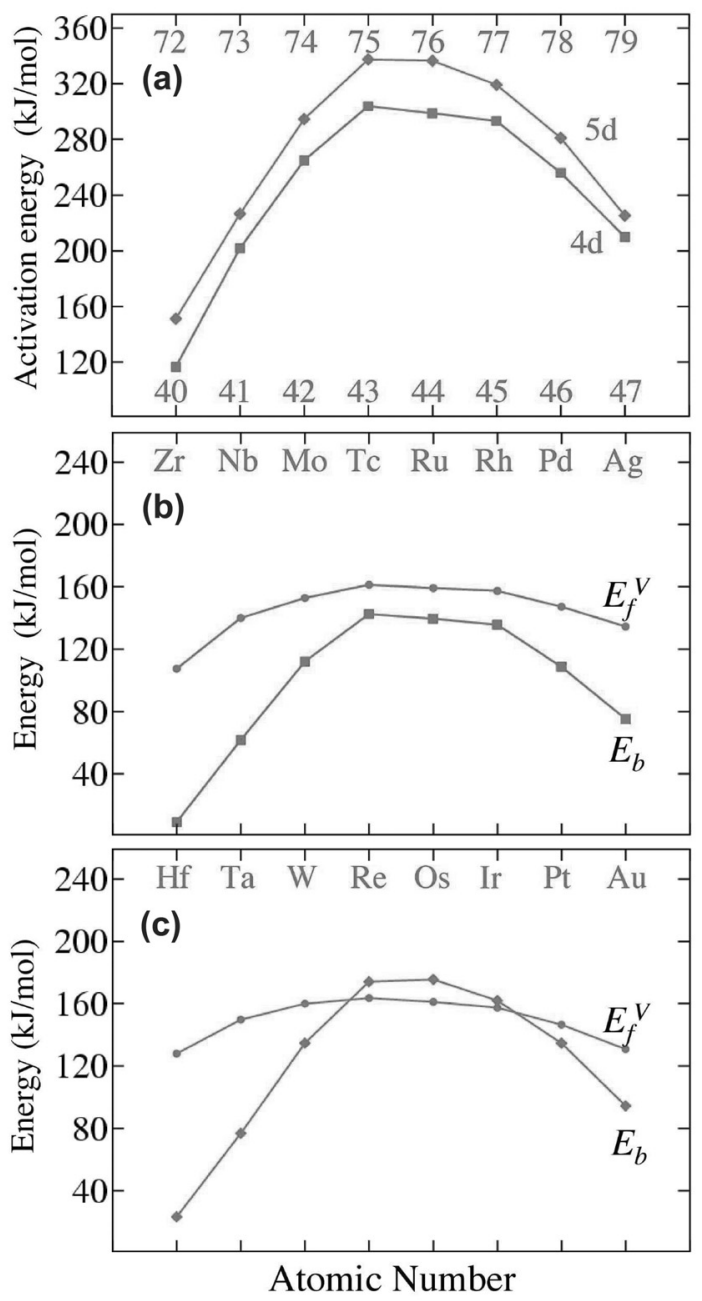

Figure 4: (a) Variation of the calculated activation energies $Q$ for diffusion for the $4 \mathrm{~d}$ and $5 \mathrm{~d}$ transition metal solutes; the corresponding contributions from $E_{b}$ and $E_{f}^{V}$ are given in (b) and (c) for the $4 \mathrm{~d}$ and $5 \mathrm{~d}$ rows respectively. 

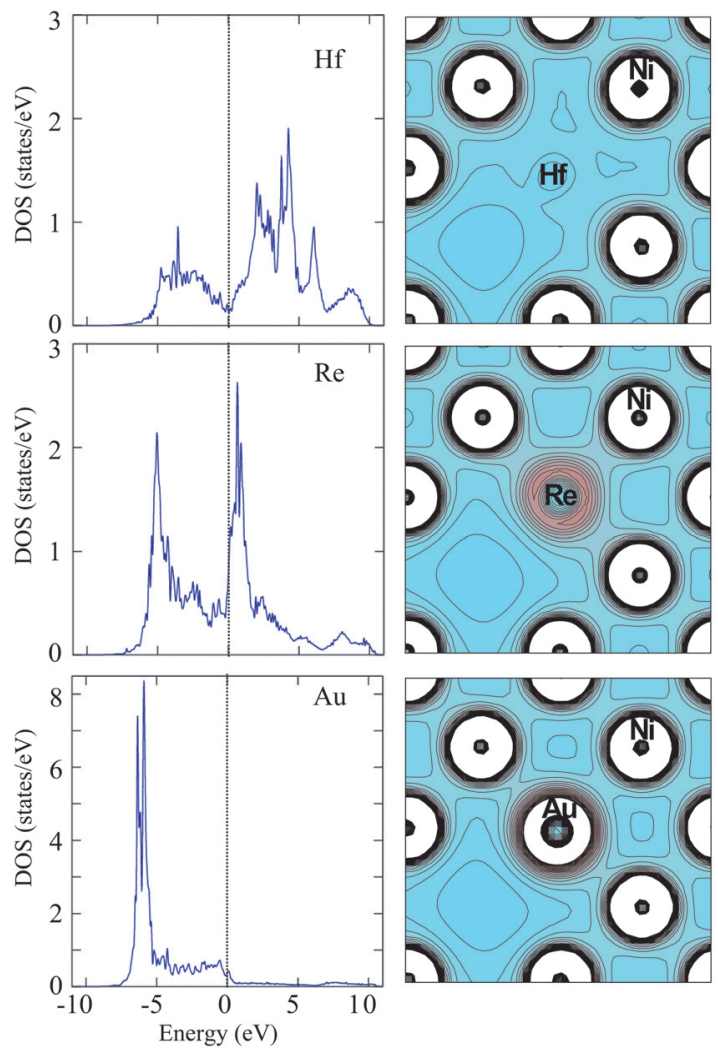

Figure 5: The valence charge density of Hf, Re and Au, representing the variation of the chemical bond across the $5 \mathrm{~d}$ row, in the $\{001\}$ plane of the $\mathrm{Ni}$ solvent, together with the corresponding variation of the electronic density of states with energy. The zero in the left hand panel corresponds to the Fermi energy.

unity, so that any possible effects of correlation are ignored. As will be shown in the last section of the paper, a direct comparison of theory and experiment is then not strictly valid. The calculations shed light on the reasons for the differing behaviour of the solutes. Consider Figure 5 which shows the valence charge densities surrounding each of Hf, Re and Au atoms which are representative of the variation of chemical bonding across the $5 \mathrm{~d}$ row. In each case, a section on the $\{001\}$ plane is shown with a solute atom at centre and a vacant neighbouring site. The corresponding variations of the elec- tronic density-of-states with energy are given in the left hand panel of Figure 5. The calculations demonstrate that (i) the d-electrons of Hf do not participate in direct bonding with the neighbouring $\mathrm{Ni}$ atoms (ii) the charge density of Re exhibits a preference along $<110>$, indicating the development of a directional bond and (iii) the d-electrons of $\mathrm{Au}$ are highly localised and chemically inactive - as confirmed by the narrow d-band and the spherical charge distribution. The variation of the $\mathrm{Ni}$-solute bond as one crosses the $4 \mathrm{~d}$ and $5 \mathrm{~d}$ transition metal rows is thus in many ways analogous to the different bonding characteristics of the pure transition metals - for the mid-row $4 \mathrm{~d}$ and $5 \mathrm{~d}$ solutes one has a significant attractive partial pressure due to the directional bonding developed within the d-states, balanced by a repulsive partial pressure arising from the contribution
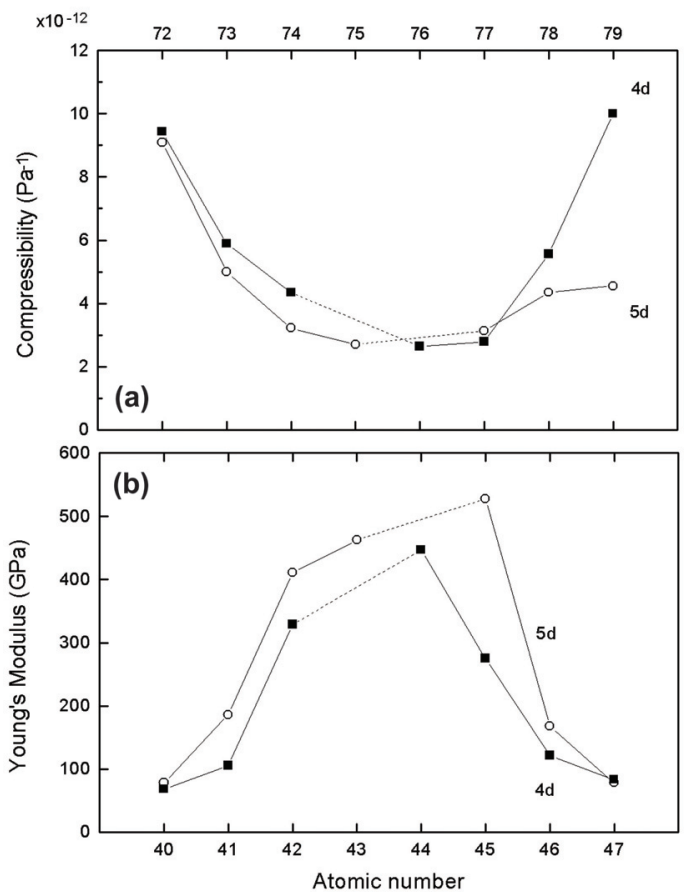

Figure 6: Variation of the bulk elastic moduli [20] of the 4d and $5 \mathrm{~d}$ transition metals with atomic number: (a) compressibility (inverse bulk modulus), and (b) Young's modulus. 
from the s-electrons. Consequently, the equilibrium Ni$\mathrm{X}$ interatomic distance is amongst the smallest when $\mathrm{X}=\mathrm{Re}$, just as elements such as Re display an atomic volume which is amongst the smallest in the $5 \mathrm{~d}$ row, the least great compressibility and the greatest Young's modulus - see Figure 6. Similar arguments apply for the $4 \mathrm{~d}$ row. These considerations make it clear that elements such as Re resist diffusion because of the nature of their bonding with the solvent $\mathrm{Ni}$, which is such as to make the solute-vacancy exchange difficult. Hence it is the Ni-solute bonding characteristics which are of importance in controlling the rate of diffusion.

That the misfit strain between the solute and solvent is not a major contributing factor can be confirmed in the following way. The contraction in the nearest neighbour nickel-solute distance as the solute atom migrates into a vacancy via the saddle point position, see Figure 3, falls out of the calculations. Only a very weak dependence on the size of the solute is found - the contraction is $6.8 \%$

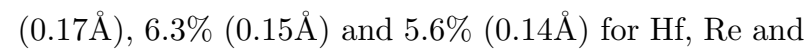
Au respectively. Therefore, the size of the solute atom and thus the lattice misfit has little effect on the energy barrier $E_{b}$.

\section{Results: 3d Row}

The results presented so far indicate that the origin of the higher diffusion energy barriers for the smaller $4 \mathrm{~d}$ and $5 \mathrm{~d}$ solute atoms is the development of directional dbonding. It is interesting to examine whether the same trends and mechanisms apply to the diffusion of the $3 \mathrm{~d}$ solutes in Ni. One should note however that there are two principal differences between the $3 \mathrm{~d}$ and $4 \mathrm{~d} / 5 \mathrm{~d}$ solutes. First, the size misfit with the Ni host is less pronounced for the $3 \mathrm{~d}$ solutes. Second, the effect of magnetism - which is intrinsic to the $3 \mathrm{~d}$ transition metals adds complexity to the electronic structure. Thus one is no longer treating the diffusion of very much larger solute atoms in a smaller lattice, as before, with more subtle effects likely to be present. For example, it is well known that the effect of magnetism changes the char- acteristics of chemical bonds; this should be reflected in the calculated barrier energies $E_{b}$ of the $3 \mathrm{~d}$ solutes. Indeed, our calculations reveal that the activation energies for diffusion of the $3 \mathrm{~d}$ solutes display a complex and intriguing dependence on the atomic number, see Figure 7. As before, the solute-vacancy binding energy $E_{f}^{V}$ exhibits a weaker dependence on the atomic number than the diffusion barrier $E_{b}$. However, very unlike the case of the $4 \mathrm{~d}$ and $5 \mathrm{~d}$ solutes, a local minimum in the diffusion energy barrier profile is predicted to exist in the middle of the $3 \mathrm{~d}$ row, at the position corresponding to Mn. Most significantly, the existence of a local minimum in the diffusion energy barrier is accompanied by the occurrence of a maximum in the magnetic moment

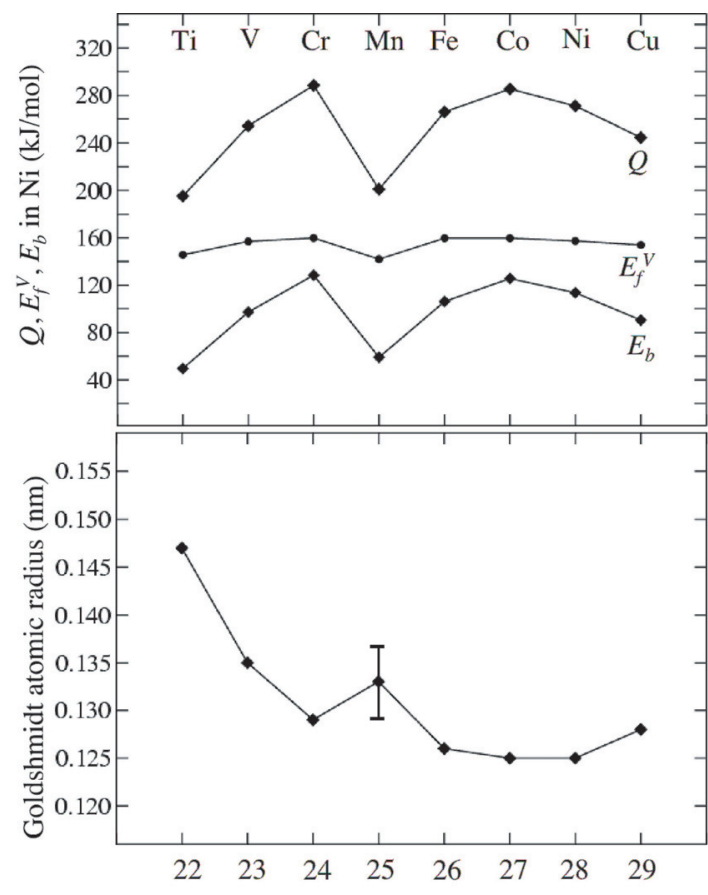

Atomic Number Z

Figure 7: (a) Variation of the calculated activation energy $Q$ for the diffusion of the $3 \mathrm{~d}$ solutes in $\mathrm{Ni}$, and the contributions from the barrier energy $E_{b}$ and the vacancy formation energy $E_{f}^{V}$, (b) variation of the Goldschmidt atomic radius of the $3 \mathrm{~d}$ solutes with atomic number, illustrating the anomalous behaviour of $\mathrm{Mn}$. 


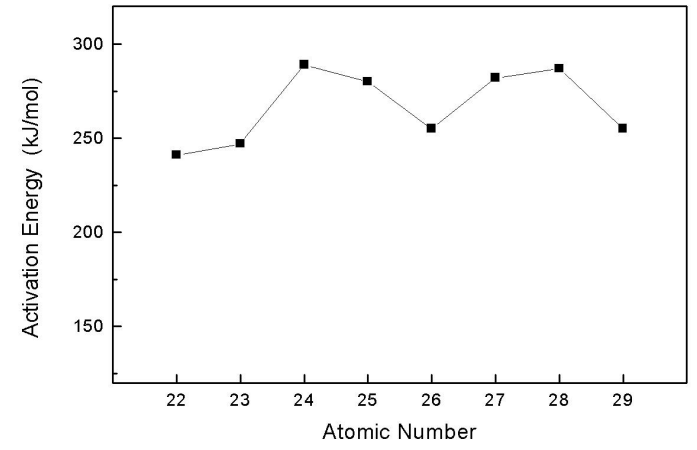

Figure 8: Variation of the experimental values [8-14] of the activation energy of interdiffusion for the $3 \mathrm{~d}$ transition metals with nickel. Note that the correlation factor is taken to be unity.

for the $3 \mathrm{~d}$ row. It is found that $\mathrm{Mn}$ has the largest magnetic moment of the $3 \mathrm{~d}$ solute atoms: the magnetic moments (evaluated within the Goldschmidt radii) are $-0.4 \mu_{B},-1.2 \mu_{B}, 3.1 \mu_{B}, 2.7 \mu_{B}$, and $1.7 \mu_{B}$ for the $\mathrm{V}$, $\mathrm{Cr}, \mathrm{Mn}, \mathrm{Fe}$, and Co solutes, respectively. Note here that the negative sign indicates that the moment is coupled antiparallel to the moment of the nearest Ni neighbour. These results demonstrate that magnetism plays a central role in determining the magnitude of the energy barrier for diffusion.

In the presence of the magnetic exchange interaction, the d-band of the $3 \mathrm{~d}$ solutes is split into majority-spin and minority-spin channels. From $\mathrm{Cr}$ to $\mathrm{Mn}$, the results indicate that there is a change in the magnetic coupling between the solutes and neighbouring $\mathrm{Ni}$ atoms - indicating an abrupt change in the d-bonding characteristics. In the case of $\mathrm{Mn}$, the majority-spin d-states are nearly fully occupied and do not participate actively in the chemical bonding; on the other hand, the minorityspin d-states are less than half-filled and the occupied states do not have the $\langle 110\rangle$ directional d-bonding components. Thus, in the absence of $<110>$ directional bonds, the Mn solutes are easy to compress so that the resulting energy barrier for diffusion is small. For Fe, the minority-spin d-states with the directional bonding characteristic become progressively occupied, which accounts for Fe's energy barrier $E_{b}$ being greater than for that of Mn. Interestingly, these results are consistent with the 'anomalous' trends in both the atomic radius and compressibility for the $3 \mathrm{~d}$ transition metals; in particular, the compressibility of Mn greatly exceeds that of its adjacent atoms in the periodic table. Due to its complex structure, the reported Goldschmidt radius of $\mathrm{Mn}$ varies considerably in the literature and an error bar represents the values in Figure 7. Again, a correlation seems to exist between the diffusion activation energy and the solute atomic size for $3 \mathrm{~d}$ solutes, in the same way as for $4 \mathrm{~d}$ and $5 \mathrm{~d}$ solutes.

In Figure 8, the experimentally determined values [814] of the apparent activation energy for diffusion $Q$ are plotted for the $3 \mathrm{~d}$ transition metals - derived by plotting $\ln \{D\}$ vs $1 / T$ with the correlation factor $f$ assumed to be unity. It is notable that the variation with atomic number of the experimental values of $Q$ determined in this way is not as strong for the $3 \mathrm{~d}$ row as for the $4 \mathrm{~d}$ and 5 d rows, see Figure 2 - and that the values are consistently smaller than for the $4 \mathrm{~d}$ and $5 \mathrm{~d}$ solutes. Of interest is the local minima which occurs at $\mathrm{Fe}-$ a feature which is absent for the $4 \mathrm{~d}$ and $5 \mathrm{~d}$ solutes. The predicted variation of $Q$ for the 3 d solutes - see Figure $7 \mathrm{a}$ - shows some similarity with the experimental one, although as

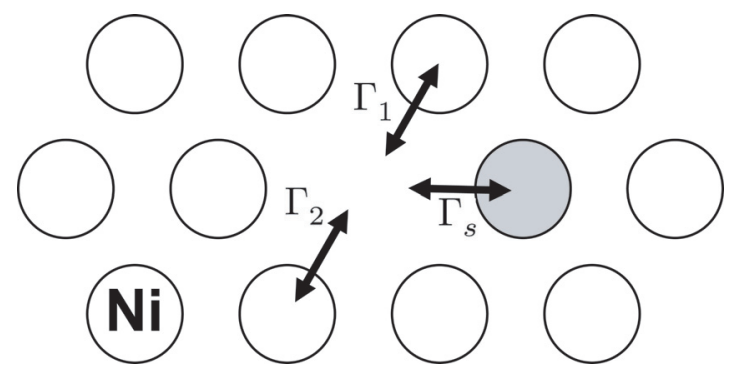

Figure 9: Illustration of the meanings of the exchange frequencies $\Gamma_{s}, \Gamma_{1}$ and $\Gamma_{2}$ which correspond to the rate of solute/vacancy exchange, and the rates of rotation and dissociation of the solute/vacancy pairs respectively. 
before the reader is cautioned that a direct comparison of theory and experiment is hampered by the assumption that $f=1$. As it turns out, it is important to account for correlation effects as will be demonstrated in the following section.

\section{Discussion: Correlation Effects}

For the accurate prediction of the diffusion rates using these methods, it has been discovered that it is necessary to take account of correlation effects. The correlation factor in Equation 1 acknowledges the possibility that a diffusing atom can jump back to its previous lattice site if the solute-vacancy pair remains un-dissociated. It can be evaluated from Lidiard's five-frequency exchange model $[15,16]$ according to

$$
f=\frac{2 \Gamma_{1}+7 \Gamma_{2}}{2 \Gamma_{s}+2 \Gamma_{1}+7 \Gamma_{2}}
$$

where $\Gamma_{1}$ and $\Gamma_{2}$ are the frequencies of exchange between a vacancy and a nickel atom which results in rotation and dissociation of a solute-vacancy pair respectively, and $\Gamma_{s}$ is the rate of exchange of vacancy and solute, see Figure 9. The site-exchange rates $\Gamma_{1}, \Gamma_{1}$ and $\Gamma_{s}$ can be calculated via expressions such as Equation 2, once the corresponding diffusion barriers have been evaluated using the first principles methods.

To illustrate the important influence of the correlation effects and because of the importance of Re as an alloying addition, attention is focussed here on the behaviour of the $5 \mathrm{~d}$ transition metals. For early and late $5 \mathrm{~d}$ solutes such as $\mathrm{Hf}$ and $\mathrm{Au}$, the calculations predict that $\Gamma_{s} \gg \Gamma_{2}>\Gamma_{1}$. In fact, the data indicate that the energy barrier in the $\Gamma_{2}$ term differs insignificantly (to within $10 \mathrm{~kJ} / \mathrm{mole}$ ) from that of Ni self-diffusion, and is about $40 \mathrm{~kJ} /$ mole lower than that in the $\Gamma_{1}$ term. The calculated correlation factors are then of the order of $10^{-3}$ for $\mathrm{Hf}$ and $10^{-1}$ for $\mathrm{Au}$ at $1000^{\circ} \mathrm{C}$, so that in these cases the correlation effect acts to reduce the diffusion coefficients quite substantially. On the other hand, $\Gamma_{1} \simeq \Gamma_{2} \gg \Gamma_{s}$ for mid-row solutes such as Re, so that $f \simeq 1$. Thus the rate-determining step for diffusion in this case is the exchange of the solute-vacancy pair.

The computed diffusion coefficients of the $5 \mathrm{~d}$ elements and their variation with atomic number are given in Figure $10 \mathrm{a}$ - with the correlation effect ignored and therefore $f=1$ in all cases - and in Figure 10b with the correlation factor included properly. For the mid-row elements, the correlation effect has little influence on the predicted values of the diffusion coefficients, but for elements to the far west or far east of the $5 \mathrm{~d}$ row its
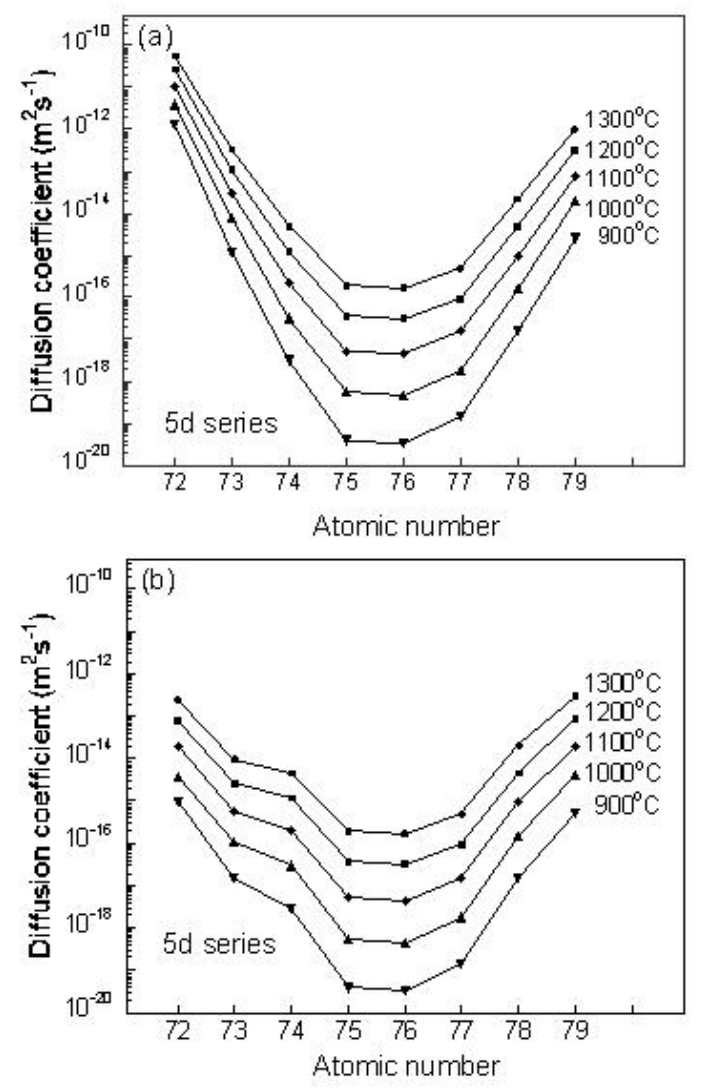

Figure 10: Predicted variation of the diffusion coefficient of the 5 d elements in nickel: (a) without the correlation effect accounted for, and (b) with the correlation effect included. The predictions in (b) should be compared with the experimental data in Figure 1b. 
effect is quite marked. With the correlation factor included in the calculations, the predicted values are in reasonable agreement with the experimental data given in Figure 1. Moreover, it has been found that the predicted diffusion coefficients for the $3 \mathrm{~d}$ solutes are in reasonable agreement with the experimental values if the correlation effect is included - due to space limitations the full details will be given elsewhere.

\section{Conclusions}

First principles electronic structure calculations, using the local density functional method, have been used to study the diffusion of the transition metals in nickel. From a practical standpoint, our most significant results relate to the $4 \mathrm{~d}$ and $5 \mathrm{~d}$ elements. Rates of diffusion are slowest for elements at the centre of the transition metal block, e.g. Re and Ru, despite their having atomic sizes which are the smallest and closest to nickel. Conversely, elements at the far east or west of the rows diffuse the fastest, although they possess the largest atomic radii. These theoretical results are consistent with recent experimental data. The variation of the diffusion coefficient with atomic number is largely due to differences in the barrier energy for diffusion along $<110>$, i.e. for solute-vacancy exchange, and must be attributed to differing characteristics of the bonding. Hence the size effect - implying a size misfit - is not so important; instead it is the electronic structure which causes these phenomena. Dense elements such as Re and Ru possess small atomic radii and their compressibilities are amongst the least great of all the transition metals; alloying thus causes directional and incompressible Ni-Re and $\mathrm{Ni}-\mathrm{Ru}$ bonds, which do not favour solute-vacancy exchanges. This effect dominates over any influences of atomic radius, misfit strain and some small differences in the vacancy-solute binding energy. It is very likely that these results go a long way to explaining the beneficial effects conferred by alloying additions such as Re. They also point to ways in which the creep resistance of single crystal superalloys might be further improved.

\section{Acknowledgements}

Helpful discussions with Dr C.T. Liu are greatly appreciated. The research of C.L.F., A.J., and M.K. was sponsored by the Division of Materials Sciences \& Engineering, U.S. Department of Energy, under contract DE-AC05-00OR-22725 with UT-Battelle, LLC. This research (A.J. and M.K.) was supported in part by an appointment to the Oak Ridge National Laboratory Postdoctoral Research Associates Program, which is administered jointly by the Oak Ridge Institute of Science and Education and Oak Ridge National Laboratory. R.C.R. acknowledges funding from the Natural Sciences \& Engineering Research Council of Canada (NSERC). Ainul Akhtar and Jacky Cheng of UBC are acknowledged for their help with some of the figures for this paper.

\section{References}

1. T.M. Pollock and A.S. Argon, Acta metall. mater., 40, 1, (1992).

2. N. Matan, D.C. Cox, C.M.F. Rae and R.C. Reed, Acta mater., 47, 2031, (1999).

3. W.S. Walston, J.C. Schaeffer and W.H. Murphy, in 'Superalloys 1996', edited R.D. Kissinger, D.J. Deye, D.L. Anton, A.D. Cetel, M.V. Nathal, T.M. Pollock and D.A. Woodford, 9, The Minerals, Metals and Materials Society, Warrendale, PA, USA, (1996).

4. J.M. Smialek and G.M. Meier, in 'Superalloys II: High Temperature Materials for Aerospace and Industrial Power', C.T. Sims, N.S. Stoloff and W.C. Hagel (eds), John Wiley \& Sons, New York, USA, 293, (1987).

5. M.S.A. Karunaratne and R.C. Reed, Acta mater., 51, 2905, (2003).

6. M.S.A. Karunaratne, $\mathrm{PhD}$ Thesis, Univ of Cambridge, (2001).

7. A. Janotti, M. Krcmar, C.L. Fu and R.C. Reed, Ac- 
cepted for publication in Physical Review Letters.

8. N. Komai, M. Watanabe, Z. Horita, T. Sano and M. Nemoto, Acta mater., 46, 4443, (1998).

9. A. Davin, V. Leroy, D. Coutsouradis \& L. Habraken, Cobalt, 19, 51 (1963).

10. S.B. Jung, T. Yamane, Y. Minamino, K. Hirao, H. Araki and S. Saji, J Mater Sci Letters, 11, 1333, (1992).

11. R.A. Swalin and A. Martin, Trans AIME, 206, 567, (1956).

12. T. Ustad and H. Sorum, Phys Stat Sol., 20, 285, (1973).

13. M.S. Anand, S.P. Murarka and R.P. Agarwala, J of Appl Phys., 36, 3860, (1965).
14. B. Jonsson, Scandinavian Journal of Metallurgy, 24, 21, (1995).

15. A.R. Allnatt and A.B. Lidiard, Atomic Transport in Solids, Cambridge University Press, Cambridge (1993).

16. M.E. Glicksman, Diffusion in Solids, Wiley, New York (2000).

17. W. Kohn and L.J. Sham, Phys Rev, 140, A1133, (1965).

18. D. Vanderbilt, Phys Rev B, 41, 7892, (1990).

19. G. Kresse and J. Furthmuller, Phys Rev B, 54, 11169, (1996).

20. G.W.C. Kaye and T.H. Laby, 'Tables of Physical and Chemical Constants', 14th Edition, Longman, London, United Kingdom, (1982). 